\title{
A FUNCTION WHICH IS ARC-ANALYTIC BUT NOT CONTINUOUS
}

\author{
E. BIERSTONE, P. D. MILMAN, AND A. PARUSIŃSKI
}

(Communicated by Clifford J. Earle, Jr.)

\begin{abstract}
We construct an arc-analytic function (i.e., a function analytic on every real analytic arc) which is not continuous, as well as a continuous arcanalytic function which is not subanalytic.
\end{abstract}

A function $f(x)$ of $n$ real variables $x=\left(x_{1}, \ldots x_{n}\right)$ is called arc-analytic if $f(x(t))$ is analytic for every real analytic arc $x=x(t)$. In this note, we construct:

(1) an arc-analytic function $f\left(x_{1}, x_{2}\right)$ which is not continuous; and

(2) a continuous arc-analytic function $f\left(x_{1}, x_{2}\right)$ whose graph is not subanalytic.

These examples answer questions raised in [1]. According to [1, Theorem 1.4], $f\left(x_{1}, \ldots, x_{n}\right)$ is arc-analytic and has subanalytic graph if and only if it can be transformed to an analytic function by composition with finite sequences of local blowings-up in $x$ with smooth centres. Our example (2) shows that "subanalytic" is not a superfluous hypothesis in this result. Every arc-analytic function with subanalytic graph is continuous.

The idea of our construction is the following: Let $Z_{0}=\mathbb{R}^{2}$, and fix a sequence of blowings-up $\pi_{i}: Z_{i} \rightarrow Z_{i-1}$ with one-point centres. For simplicity, assume that these centres are determined by the germ at the origin of the curve $\gamma_{0}(t)=$ $(t, 0) \in \mathbb{R}^{2}$. This means that $\pi_{1}: Z_{1} \rightarrow \mathbb{R}^{2}$ is the blowing-up of the origin and, if $\gamma_{i}$ denotes the strict transform of $\gamma_{i-1}$ by $\pi_{i}$, then $\pi_{i+1}: Z_{i+1} \rightarrow Z_{i}$ is the blowing-up with centre $\gamma_{i}(0)$. Take a sequence of arc-analytic funitons $H_{n}$ such that each $H_{n}$ becomes analytic after exactly $n$ blowings-up; i.e., $H_{n} \circ \pi_{1} \circ \cdots \circ \pi_{i}$ is analytic if and only if $i \geq n$. Then $H=\sum_{n=1}^{\infty} a_{n} H_{n}$, where $\left\{a_{n}\right\}$ is a rapidly decreasing sequence of positive numbers, is a candidate for a function which is arc-analytic but not subanalytic.

Received by the editors April 13, 1990.

1980 Mathematics Subject Classification (1985 Revision). Primary 32B20; Secondary 32B30.

The first author's research was partially supported by NSERC operating grant OGP 0009070. The second author's research was partially supported by NSERC operating grant A8849. The third author was supported by Alexander von Humboldt Stiftung. 
Consider, for example,

$$
H_{n}(x, y)= \begin{cases}x h_{n}(x, y), & (x, y) \neq 0 \\ 0, & (x, y)=0\end{cases}
$$

where

$$
h_{n}(x, y)=x^{2 n} /\left(x^{2 n}+y^{2}\right), \quad(x, y) \neq 0,
$$

$n=1,2, \ldots$ It is easy to check that $h_{n}$ is analytic (i.e., extends to be analytic) on every analytic arc and, moreover, that $h_{n}$ becomes analytic after exactly $n$ blowings-up. However, on the line $\{x=c\}, h_{n}$ restricts to $\phi_{n}(y)=$ $c^{2 n} /\left(c^{2 n}+y^{2}\right)$. If $0<|c|<1$, then the complex roots of the denominators tend to 0 as $n \rightarrow \infty$, so that $\sum_{n=1}^{\infty} a_{n} \phi_{n}(y)$ cannot converge to an analytic function. The problem here is that the complex zero sets of the denominators $f_{n}(x, y)=x^{2 n}+y^{2}$ become closer and closer to the real plane as $n \rightarrow \infty$.

We will modify the $f_{n}$ in such a way that their complex zeros do not change much as $n$ increases; in particular, the distances from their complex zero sets to a nonzero point $\left(x_{0}, y_{0}\right) \in \mathbb{R}^{2}$ will be bounded away from zero.

We first define a sequence of polynomials $g_{n}(x, y)$ inductively by the formulas

$$
\begin{aligned}
& g_{1}=f_{1}, \\
& g_{n}=\delta_{n} f_{n} g_{1}^{n-2} g_{2}^{n-3} \cdots g_{n-2}^{1}+g_{n-1}^{2}, \quad n=2,3, \ldots,
\end{aligned}
$$

where the $\delta_{n}>0$ is specified below. Then we set

$$
h_{n}(x, y)=x^{2^{n}} / g_{n}(x, y), \quad n=1,2 \ldots .
$$

We describe how the $f_{n}$ and $g_{n}$ transorm by the blowings-up $\pi_{i}$ : To begin with, $Z_{1}$ is covered by 2 coordinate charts $U_{1}$ and $U_{1}^{\prime}$, each isomorphic to $\mathbb{R}^{2}$, in which $\pi_{1}$ is given by the following formulas:

$$
\begin{aligned}
& \pi_{11}=\pi_{1} \mid U_{1}:\left(x_{1}, y_{1}\right) \mapsto\left(x_{1}, x_{1} y_{1}\right), \\
& \pi_{12}=\pi_{1} \mid U_{1}^{\prime}:\left(x_{1}^{\prime}, y_{1}^{\prime}\right) \mapsto\left(x_{1}^{\prime} y_{1}^{\prime}, y_{1}^{\prime}\right) .
\end{aligned}
$$

Therefore

$$
\begin{aligned}
& f_{n} \circ \pi_{11}=x_{1}^{2} f_{n-1}, \quad n>1, \\
& f_{n} \circ \pi_{12} \sim y_{1}^{\prime 2}
\end{aligned}
$$

(where $g \sim h$ means that $g$ equals $h$ times a factor that vanishes nowhere). Thus the strict transform $f_{1 n}$ of $f_{n}$ by $\pi_{1}$ vanishes only in $U_{1}$. (The strict transform, which is defined locally up to an invertible factor, is given in $U_{1}$ by $f_{n} \circ \pi_{11} / x_{1}^{2}$ and in $U_{1}^{\prime}$ by $f_{n} \circ \pi_{12} / y_{1}^{\prime 2}$.) Proceeding inductively, we see that for each $i=1,2, \ldots, Z_{i}$ has one chart $U_{i}=\mathbb{R}^{2}$ with coordinates $\left(x_{i}, y_{i}\right)$ such that:

(1) $\pi_{i}\left(U_{i}\right) \subset U_{i-1}$ and $\pi_{i} \mid U_{i}$ is given by

$$
\left(x_{i-1}, y_{i-1}\right)=\left(x_{i}, x_{i} y_{i}\right), \quad i=2,3, \ldots
$$


(2) The strict transform $f_{i n}$ of $f_{n}$ by $\pi_{1} \circ \pi_{2} \circ \cdots \circ \pi_{i}$ vanishes only in $U_{i}$, and

$$
\begin{aligned}
& f_{i n} \mid U_{i}=f_{n-i}, \quad n>i, \\
& f_{i n} \mid U_{i} \sim 1, \quad n \leq i .
\end{aligned}
$$

Lemma. The functions $g_{n}$ vanish only at the origin. For each $n=2,3, \ldots$, the function $g_{n}$ has multiplicity $2^{n-1}$ at 0 . ( $g_{1}$ has multiplicity 2 at 0 .) For each $n$, the strict transform $g_{i n}$ of $g_{n}$ by $\pi_{1} \circ \cdots \circ \pi_{i}$ vanishes only at the origin of $U_{i}$, and on $U_{i}$,

$$
g_{\text {in }} \sim 1, \quad n \leq i,
$$

$g_{i, i+1}$ has a nondegenerate critical point at 0 , and if $n>i+1$, then

$$
g_{i n}=\delta_{n} f_{i n} g_{i 1}^{n-2} g_{i 2}^{n-3} \cdots g_{i, n-2}^{1}+g_{i, n-1}^{2},
$$

so that $g_{\text {in }}$ has multiplicity $2^{n-i-1}$ at 0 .

Proof. By induction on $n$.

From the lemma, on $U_{1}$ we get

$$
h_{n} \circ \pi_{1}=\left(x^{2^{n}} / g_{n}\right) \circ \pi_{1} \sim x_{1}^{2^{n-1}} / g_{1 n},
$$

and, in general, on $U_{i}$ we obtain

$$
h_{n} \circ \pi_{1} \circ \cdots \circ \pi_{i} \sim x_{i}^{2^{n-i}} / g_{\text {in }}, \quad n>i .
$$

Proposition. If the $\delta_{n}$ are sufficiently small and $\left\{a_{n}\right\}$ is a rapidly decreasing sequence of positive numbers, then $h=\sum_{n=1}^{\infty} a_{n} h_{n}$ is analytic on every analytic arc, and

$$
H(x, y)= \begin{cases}x h(x, y), & (x, y) \neq 0, \\ 0, & (x, y)=0,\end{cases}
$$

defines an arc-analytic function.

Proof. We will show that the $\delta_{n}$ can be chosen so that, if $\left\{a_{n}\right\}$ is rapidly decreasing, then $h \circ \pi_{1} \circ \cdots \circ \pi_{i}$ is analytic on $Z_{i}-\left\{\gamma_{i}(0)\right\}, i=1,2, \ldots$ This guarantees that $h$ is analytic on any analytic arc except perhaps $\gamma_{0}$. On the other hand, $g_{n}(x, 0)=x^{2^{n}} g_{n}(1,0)$, where $g_{n}(1,0)>0, n=1,2, \ldots$, so that $h(x, 0)$ is constant on $\gamma_{0}$. Therefore $H$ is an arc-analytic function.

Let $X_{n}$ denote the complex zero set of $g_{n}, n=1,2, \ldots$. If we choose $\delta_{2}$ small enough, we can make $X_{2}$ close to $X_{1}$; then choosing $\delta_{3}$ small enough, we can make $X_{3}$ chose to $X_{2}$, etc. More precisely: For each $k=1,2, \ldots$, put

$$
V_{k}=\left\{(x, y) \in \mathbb{R}^{2}: 1 / k^{2}<x^{2}+y^{2}<1\right\}
$$

and

$$
c_{k}=\frac{1}{2} d\left(V_{k}, X_{1}\right), \quad k=1,2, \ldots
$$

( $d$ denotes the Euclidean distance). Choose the $\delta_{n}$ successively, small enough so that $d\left(V_{k}, X_{n}\right) \geq c_{k}$ for all $k=1, \ldots, n, n=1,2, \ldots$. With such a 
choice of the $\delta_{n}$ (and with $\left\{a_{n}\right\}$ rapidly decreasing), $h$ will be analytic in each annulus $V_{k}$; hence analytic in $0<x^{2}+y^{2}<1$.

In fact, we can choose the $\delta_{n}$ so that, also for each $i=1,2, \ldots, h \circ \pi_{1} \circ \cdots \circ \pi_{i}$ is analytic in a punctured neighborhood of the point $\gamma_{i}(0)$ (i.e., of the origin in the chart $\left.U_{i}\right)$ : Let $X_{i n}$ denote the complex zero set of $g_{i n} \mid U_{i}$ for each $n>i$, $i=1,2, \ldots$. Put

$$
V_{i k}=\left\{\left(x_{i}, y_{i}\right) \in U_{i}: 1 / k^{2}<x_{i}^{2}+y_{i}^{2}<1\right\}
$$

and

$$
c_{i k}=\frac{1}{2} d\left(V_{i k}, X_{i, i+1}\right) \text {. }
$$

It suffices to choose the $\delta_{n}$ so that also $d\left(V_{i k}, V_{i n}\right) \geq c_{i k}$ for all $k=1, \ldots, n$ and all $i=1, \ldots, n$.

On the other hand, consider any coordinate chart $\tilde{U}_{i}=\mathbb{R}^{2}$ of $Z_{i}, i=$ $0,1, \ldots$, and any compact subset $K$ of $\widetilde{U}_{i}$ that does not include $\gamma_{i}(0)$. Then the strict transform $g_{i, i+1}$ of $g_{i+1}$ by $\pi_{i} 0 \cdots \circ \pi_{i}$ vanishes nowhere on a complex neighborhood $L$ of $K$ in $\mathbb{C}^{2}$. It follows that we can choose the $\delta_{n}$ also small enough that, for all such $\widetilde{U}_{i}$ and $K, i=0,1, \ldots$, the strict transorms $g_{i n}$ of the $g_{n}$ by $\pi_{1} \circ \cdots \circ \pi_{i}$ vanish nowhere on a fixed complex neighborhood of $K$, for all $n>i$; therefore (if $\left\{a_{n}\right\}$ is rapidly decreasing) $h \circ \pi_{1} \circ \cdots \circ \pi_{i}$ converges to an analytic function in a neighborhood of $K$.

To sum up, the $\delta_{n}$ can be chosen so that, if $\left\{a_{n}\right\}$ is rapidly decreasing, then $h \circ \pi_{1} \circ \cdots \circ \pi_{i}$ is analytic on $Z_{i}-\left\{\gamma_{i}(0)\right\}, i=0,1,2, \ldots$.

Example (2). Take $H$ as in the proposition above. Clearly, $H$ is continuous. It is easy to see that each $h_{n} \circ \pi_{1} \circ \cdots \circ \pi_{i}$ is analytic precisely when $i \geq n$, and the directional derivative of $\sum_{n>i} a_{n} h_{n} \circ \pi_{1} \circ \cdots \circ \pi_{i}$ at 0 in the direction $(x, y)$ equals $a_{i+1} x^{2} / Q_{i}(x, y)$, where $Q_{i}$ is a nondegenerate quadratic form $\left(Q_{i}\right.$ is the initial form of $\left.g_{i, i+1}\right)$. Therefore, $H \circ \pi_{1} \circ \cdots \circ \pi_{i}$ is never analytic. It follows from [1, Theorem 1.4] that the graph of $H$ is not subanalytic.

Example (1). Take $h$ as in the proposition. Clearly,

$$
g_{n}(x, y)>g_{n}(x, 0)=x^{2^{n}} g_{n}(1,0),
$$

where $g_{n}(1,0)>0$, for all $x$, all $y \neq 0$, and $n=1,2, \ldots ;$ therefore,

$$
h_{n}(x, y)<h_{n}(x, 0)=1 / g_{n}(1,0)=h_{n}(1,0) \text {, }
$$

for all $x \neq 0, y \neq 0$, and $n=1,2, \ldots$. Put

$$
f(x, y)= \begin{cases}y e^{2 /\left(x^{2}+h(1,0)-h(x, y)\right)}, & (x, y) \neq 0, \\ 0, & (x, y)=0 .\end{cases}
$$

We claim that $f(x, y)$ is an arc-analytic function but that, if $\left\{a_{n}\right\}$ is sufficiently rapidly decreasing, then

$$
\lim _{x \rightarrow 0} \frac{h(1,0)-h\left(x, e^{-1 / x^{2}}\right)}{x^{2}}=0,
$$


so that $\lim _{x \rightarrow 0} f\left(x, e^{-1 / x^{2}}\right)=+\infty$ and $f$ is not continuous at 0 .

Indeed, for every $n=1,2, \ldots$,

$$
0 \leq h_{n}(1,0)-h_{n}(x, y) \leq \frac{y^{2}}{x^{2^{n}} g_{n}(1,0)^{2}} \cdot \frac{g_{n}(x, y)-g_{n}(x, 0)}{y^{2}} .
$$

Since the second fraction on the right is a polynomial in $(x, y), h_{n}(1,0)-$ $h_{n}\left(x, e^{-1 / x^{2}}\right)$ is flat at 0 . Therefore, if $\left\{a_{n}\right\}$ is rapidly decreasing, $h(1,0)-$ $h\left(x, e^{-1 / x^{2}}\right)$ is flat at 0 , so that $\lim _{x \rightarrow 0}\left(h(1,0)-h\left(x, e^{-1 / x^{2}}\right)\right) / x^{2}=0$.

To show that $f(x, y)$ is arc-analytic, it suffices to prove

$$
h(1,0)-\lim _{t \rightarrow 0} h(x(t), y(t))>0,
$$

for any analytic arc $\sigma_{0}(t)=(x(t), y(t))$ such that $\sigma_{0}(0)=0$ and $y(t) \not \equiv 0$. Let $\sigma_{i}$ denote the strict transform of $\sigma_{i-1}$ by $\pi_{i}: Z_{i} \rightarrow Z_{i-1}, i=1,2, \ldots$. (In particular $\left.\sigma_{i-1}=\pi_{i} \circ \sigma_{i}.\right)$ There is a positive integer $r$ such that $\sigma_{i}(0)=\gamma_{i}(0)$, $0 \leq i<r$, and $\sigma_{r}(0) \neq \gamma_{r}(0)$. If $n>i$, then $\left(h_{n} \circ \pi_{1} \circ \cdots \circ \pi_{i}\right)(c)=0$ for all $c \in \pi_{i}^{-1}\left(\gamma_{i-1}(0)\right), c \neq \gamma_{i}(0)$ (since $h_{n} \circ \pi_{1} \circ \cdots \circ \pi_{i} \sim x_{i}^{2^{n-i}} / g_{\text {in }}$ on $\left.U_{i}\right)$. Therefore, $h_{n}(x(0), y(0))=0$ for all $n>r$, and

$$
h(1,0)-h(x(0), y(0)) \geq \sum_{n=r+1}^{\infty} \frac{a_{n}}{g_{n}(1,0)}>0 .
$$

\section{REFERENCES}

1. E. Bierstone and P. D. Milman, Arc-analytic functions, Invent. Math. 101 (1990), 411-424.

(E. Bierstone and P. D. Milman) Department of Mathematics, University of Toronto, Toronto, Ontario, Canada M5S 1A1

(A. Parusiński) Institute of Mathematics, University of Gdańsk, 80-952 Gdańsk, Poland

Current address: Department of Mathematics, University of Georgia, Athens, Georgia 30602 